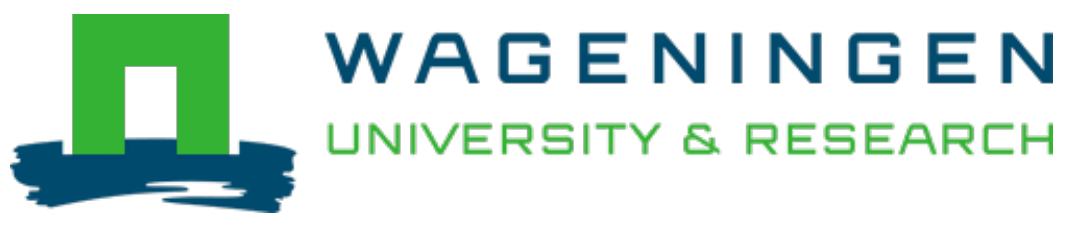

\title{
Natural and induced surface roughness determine frictional regimes in hydrogel pairs
}

\author{
Tribology International \\ Rudge, Raisa E.D.; Scholten, Elke; Dijksman, Joshua A. \\ https://doi.org/10.1016/j.triboint.2019.105903
}

This article is made publicly available in the institutional repository of Wageningen University and Research, under the terms of article $25 \mathrm{fa}$ of the Dutch Copyright Act, also known as the Amendment Taverne. This has been done with explicit consent by the author.

Article 25 fa states that the author of a short scientific work funded either wholly or partially by Dutch public funds is entitled to make that work publicly available for no consideration following a reasonable period of time after the work was first published, provided that clear reference is made to the source of the first publication of the work.

This publication is distributed under The Association of Universities in the Netherlands (VSNU) 'Article $25 \mathrm{fa}$ implementation' project. In this project research outputs of researchers employed by Dutch Universities that comply with the legal requirements of Article $25 \mathrm{fa}$ of the Dutch Copyright Act are distributed online and free of cost or other barriers in institutional repositories. Research outputs are distributed six months after their first online publication in the original published version and with proper attribution to the source of the original publication.

You are permitted to download and use the publication for personal purposes. All rights remain with the author(s) and / or copyright owner(s) of this work. Any use of the publication or parts of it other than authorised under article $25 \mathrm{fa}$ of the Dutch Copyright act is prohibited. Wageningen University \& Research and the author(s) of this publication shall not be held responsible or liable for any damages resulting from your (re)use of this publication.

For questions regarding the public availability of this article please contact openscience.library@,wur.nl 


\title{
Natural and induced surface roughness determine frictional regimes in hydrogel pairs
}

\author{
Raisa E.D. Rudge ${ }^{\mathrm{a}, \mathrm{b}}$, Elke Scholten ${ }^{\mathrm{a}}$, Joshua A. Dijksman ${ }^{\mathrm{b}, *}$ \\ ${ }^{a}$ Physics and Physical Chemistry of Foods, Wageningen University, Wageningen, the Netherlands \\ ${ }^{\mathrm{b}}$ Department of Physical Chemistry and Soft Matter, Wageningen University, Wageningen, the Netherlands
}

\section{A R T I C L E I N F O}

\section{Keywords:}

Hydrogels

Friction

Surface roughness

Asperity size

\begin{abstract}
A B S T R A C T
Hydrogels display extremely complex frictional behavior with surprisingly slippery surfaces. We measure the sliding behavior of hydrogels submerged in water using a custom-made tribotool. Samples with an imposed surface roughness give two distinct frictional regimes. Friction coefficients in the first regime change with asperity sizes and Young's moduli. Under increased normal force, a second frictional regime emerges likely due to smoothening of asperities. Friction coefficients in the second regime remain constant across length scales of roughness and appear to be material specific. The hydrogel polymer network also directly influences the surface topography, and with that, the frictional behavior of hydrogels. We highlight the tribological importance of surface roughness at different length scales, which provides potential to engineer functional frictional behavior.
\end{abstract}

\section{Introduction}

Hydrogels are chemically or physically cross-linked water absorbing networks of polymers. Hydrogels can be easily modified and thus applied in many contexts such as bio-materials [1-3] and novel food systems [4-6], as their network formation is sensitive to easily accessible tuning parameters such as polymer type, polymer concentration, $\mathrm{pH}$ and temperature. The large range of tunable material properties including porosity, hardness and water holding ability make hydrogels a material of choice for many applications. One significant feature of hydrogels is they can be very slippery [7-9]. Despite this promise, there is a general lack of fundamental understanding of the interfacial dynamics during sliding of specifically hydrogels and soft materials in general [10-12], even against ideally flat substrates [13-15]. The variety of potential applications makes understanding the emergence of friction between two hydrogel surfaces of pressing interest. More broadly, to illuminate the origin of the complex frictional behavior displayed by soft and porous solids, hydrogels are very suitable candidates to test various tribological hypotheses, precisely because their material properties such as gel strength, porosity, elasticity and surface roughness can be easily monitored and adapted.

Simple dry sliding friction between two non-porous solid materials is usually well described by Amontons-Coulombs' laws of friction [16]. The implied proportionality of $F_{F}$ and $W$ does not always hold and appears not sufficient to capture the frictional behavior of more complex systems, specifically lubricated friction between two soft solids. Mechanisms observed in hydrogel friction include attraction and repulsion between interacting surfaces, non-uniform fluid film formations and normal force dependencies $[11,17,18]$. Using hydrogels and glass substrates with varying degrees of roughness, clear differences in frictional behavior have been found for smooth and rough solid surfaces as smooth surfaces gave lower friction coefficients [19]. Previous work has shown that material friction depends also on the Young's modulus of the material [20]. Friction between soft materials is thus dependent on (bulk) contact mechanics at the hydrogel interface, but also depends on direct interactions between the two surfaces [21,22]. It is not intuitive how to apply or modify the framework used to describe dry solids to hydrogels: first, they are porous deformable solids that consist mainly of water, a lubricating fluid which could be expelled under applied loads. Second, hydrogels are soft solids and under high normal loads will thus deform substantially. [23,24]. This in turn affects the shape of the contacting surfaces and with that the contact area and pressure $[13,18]$. Additional dissipation mechanisms such as water release or even hydrogel network rearrangements or breakdown may then arise, which can add to the effective friction coefficient. In light of all the possible dissipation mechanisms that can occur, the robustness of low frictional sliding of hydrogels may seem especially surprising. Nevertheless, the slipperiness of hydrogels has been observed when paired with different materials from glass to textile [19,25-27]. The low friction dynamics remain when friction between two hydrogels in so

\footnotetext{
* Corresponding author.

E-mail address: joshua.dijksman@wur.nl (J.A. Dijksman).
} 
called "Gemini" contacts are studied $[17,18,28]$. One may naively think that Gemini contacts are relatively simple systems, yet also here friction depends on bulk and interface characteristics. For example, increasing the hydrogel mesh size by increasing the polymer concentration leads to lower friction coefficients [28]. With this same Gemini system, normal force dependence was observed: a decrease in friction coefficient was found with an increasing normal force, which scaled with $\mu \propto F_{N}^{-1 / 3}$ [18]. In a different study, with two cellulose hydrogel disks in contact, it was found that a fluid trap is created in the center of the hydrogel-hydrogel contact. As such, the lubricating fluids present strongly influenced the contact area and the frictional behavior of the hydrogels in contact [17].

Among all the complexity in the frictional dynamics in soft lubricated hydrogel contacts, we find that roughness is a surprisingly simple, yet dominant contributor to friction on soft hydrogel substrates across length scales. We measure friction dynamics between two fully submerged, water-lubricated hydrogels in a custom-3D-printed tribology set-up. The ability to tune surface roughness enables us to show that surface roughness is responsible for a normal force dependence of $\mu$, specifically the creation of two distinct frictional regimes. Additionally we show that higher friction coefficients are associated with rougher substrates, even with two highly elastic surfaces. We use molding techniques and a variety of polymer properties to create hydrogels with different roughness, ranging from extremely smooth to randomly rough. We use the natural surface structure of seemingly "flat" substrates to extend the range of surface structures we can probe to length-scales ranging from microns to millimeters. By 3D printing patterned molds, we even create regular surface patterns, and we exploit the tunability of hydrogels to check for the role of the Young's modulus. For all substrates explored we find that surface roughness enhances friction but only up to a certain level of normal forces, which we hypothesize to indicate the maximum load needed for asperity flattening. Consistent with this picture is that for samples that naturally have no asperities, we find the typical ultra-low friction dynamics, and no normal force dependence. Our results indicate that surface roughness can be relevant for materials that may appear to be flat and provides a route towards the rational control over frictional dynamics.

\section{Materials and methods}

Samples of 10, 15 and $20 \mathrm{wt} \%$ gelatin were made by slowly adding gelatin powder (gelatin from porcine skin, Type A, Sigma-Aldrich) to water at room temperature. The mixture was stirred vigorously, heated to $60{ }^{\circ} \mathrm{C}$ and left to stir for at least $30 \mathrm{~min}$, until a clear solution was obtained. Agar powder (Agar-Agar, Caldic ingredients) and water were mixed at room temperature and subsequently microwaved for approximately $30 \mathrm{~s}$ to obtain a clear $3 \mathrm{wt} \%$ agar solution. Polyacrylamide (PAAm) polymer solutions were made by mixing $20 \mathrm{wt} \%$ of acrylamide (Sigma-Aldrich) with $1 \mathrm{wt} \% \mathrm{~N}, \mathrm{~N}^{\prime}$-methylenebis(acrylamide) (SigmaAldrich), in water and slowly heating the mixture while stirring, allowing the powders to fully dissolve. The polyacrylamide mixture was then cooled down to room temperature, after which $0.05 \mathrm{wt} \%$ of the initiator, ammonium persulfate (Bio-Rad), was added. Then, $0.05 \mathrm{wt} \%$ of crosslinker $\mathrm{N}, \mathrm{N}, \mathrm{N}^{\prime}, \mathrm{N}^{\prime}$-tetramethyl ethylenediamine (TEMED, Sigma-Aldrich) was added to the aqueous mixture for chemical cross-links to form. The gelatin, agar and PAAm solutions were then poured into respective molds to prepare hydrogel probes and substrates used as tribopairs.

\subsection{Hydrogel tribopair preparation}

The (bio)polymer solutions were poured into a Petri dish with an inner diameter of $86 \mathrm{~mm}$ to obtain flat hydrogel substrates. Sample height was approximately $1.5 \mathrm{~mm}$, similar to that of the Petri dish. For the hemispherical probe, a custom-made silicone rubber mold was used (Moldstar TM 20 T, Smooth-On, Inc). After the molds were filled with the hydrogel solutions, samples were left overnight to allow the gelation process to complete. The samples remained on a level table top at room temperature during the first stages of hardening to ensure a smooth and level surface.

To chemically cross-link gelatin hydrogels, hardened samples were submersed in a $5 \mathrm{wt} \%$ glutaraldehyde solution (Sigma-Aldrich). The cross-linking process was assumed to be completed when the near colorless sample changed into a uniformly brownish orange hydrogel. Residual glutaraldehyde was removed by first rinsing the hydrogels with water and then immersing the samples overnight in a container with a large amount of water which was placed on a mechanical shaker.

Coated abrasives were used to create irregularities on the surface of the hydrogel disks. Grit labels of the sandpapers used were P2500 (Starcke USA), P400, P180, P120 and P80 (3 M), representing an average particle diameter of $8.4,35,82,125$ and $201 \mu \mathrm{m}$ respectively. The particles on a sandpaper surface can however vary greatly in diameter, height, shape and distribution on the surface. Negative molds of the sandpaper were made using Moldstar TM $20 \mathrm{~T}$ (Smooth-On, Inc). The gelatin solution was poured onto the molds and gel formation was allowed. The hygrogels were subsequently cross-linked with glutaraldehyde as described previously. After solidification, the substrate and probe were inserted in the custom-made tribotool geometry.

\subsection{Stiffness of hydrogels}

We use the Youngs modulus to quantify the ability of the hydrogel to withstand elastic deformation when subjected to a stress. The Young's modulus $(E)$ of all hydrogels was measured using samples made in a Petri dish with a diameter of $35 \mathrm{~mm}$ with a height of around $7 \mathrm{~mm}$. These compression tests are non-destructive, allowing the materials to elastically return to their original shape. Elastic behavior is also expected during our frictional measurements at relatively low normal forces (below $1 \mathrm{~N}$ ).

Samples were compressed using a $75 \mathrm{~mm}$ diameter cone-plate with a $1^{\circ}$ angle indentor on a rheometer (MCR 501, Anton Paar) at a compression rate of $50 \mu \mathrm{m} / \mathrm{s}$. During compression the force was measured as a function of deformation. Using the sample area and height, this was transformed into a stress - strain curve. This stress-strain curve typically displays nonlinear behavior at small deformation for hydrogels [2] in part due to natural sample height variation. At a strain of approximately $5 \%$ the obtained stress-strain curve becomes linear and the corresponding slope was used to calculate the Young's moduli of the hydrogels.

\subsection{Cryogenic temperature scanning electron microscopy (cryo-SEM)}

The hydrogel surfaces were visualized using cryo-SEM (FEI Magellan 400). The samples were frozen by plunge freezing using liquid nitrogen to keep them in their natural hydrated state. Due to rapid freezing, water forms amorphous ice that does not damage the material structure like crystalline ice would. The frozen samples were fractured by striking the sample with a metal rod similar to the method reported by Kim \& coworkers [29]. This allows for viewing of not only the surface, but also exposes parts of the internal (bulk) structures. Distinctly different internal and surface characteristics could be distinguished for each chemically distinct sample using this technique.

\subsection{X-ray tomography (XRT)}

For 3D imaging, XRT, a non-invasive imaging technique, was used to image surfaces with asperities from 50 to $400 \mu \mathrm{m}$. A Phoenix $\mathrm{v}$ |tome|x m (General Electric, Germany) tomographer was used for this purpose. Hydrogel samples were cut into pieces of several millimetres and inserted into an Eppendorf tube. The tube was kept closed to avoid dehydration of the hydrogel samples during measurements. Hydrogel samples were scanned over a rotation of $360^{\circ}$ and analyzed using Avizo software for image reconstruction and visualization post processing. 


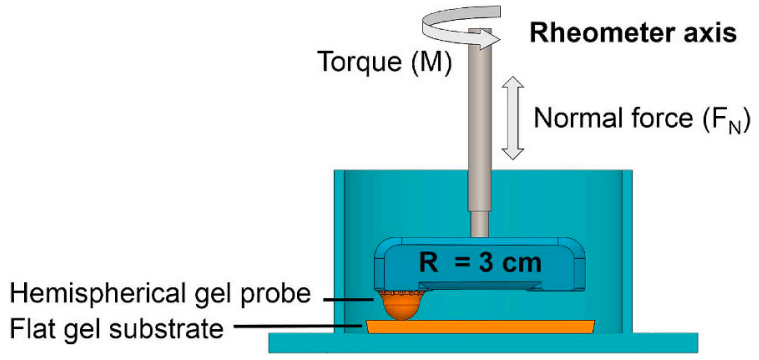

Fig. 1. Cross-sectional illustration of the gel-on-gel tribotool. The hemispherical probe is inserted into the holder and is moved alongside the hydrogel substrate using the rheometer axis. The distance between the rheometer shaft and the center of the probe is $3 \mathrm{~cm}$. The diameter of the hemispherical probe is $2 \mathrm{~cm}$.

\subsection{Frictional measurements}

The gel-on-gel tribotool used in our measurements (Fig. 1) was made using a 3D printer (Stratasys Objet30, Scholar, RGD240 plastic) and the geometry is also described more briefly elsewhere [30]. The upper part of our custom-made tribotool consists of an arm $(3 \mathrm{~cm})$ with a cavity, for insertion of the hemispherical hydrogel probe. This component was installed on a rheometer (MCR 501, Anton Paar). The rheometer is able to rotate the arm and move the arm vertically. The lower component of the tribotool is a cylindrical holder for a flat, disk-shaped hydrogel substrate. The hydrogel disk was fastened in the container using a ring-shaped clamp to avoid movement of the hydrogel substrate during measurements. This container was filled with water covering both the substrate and the upper part of the arm. The hydrogel probe attached to the arm was rotated at preset angular velocities between 0.005 and 0.1 revolutions per second (rps) corresponding to sliding velocities of $1-20 \mathrm{~mm} / \mathrm{s}$. Measurements started after the value of the normal force was reset to zero on the rheometer and the zero gap was set as a reference distance between the probe and the substrate. The initial point of contact between the hydrogels was determined by constructing a force - distance curve. The slightest increase in normal force was taken as the first contact point. From the torque $(M)$ measured by the rheometer we calculate the friction force as $F_{F}=M / R(R=0.03 \mathrm{~m})$.

Frictional measurements were performed with alternating "rotational" and "indenting" intervals. The first measurement interval consists of rotation of the probe without the probe and substrate being in contact, while the probe is completely submerged in water. This measurement provides the background signal to correct for the torque needed to move the arm at a given angular velocity in the fluid inside the measuring cup. After this step, the probe is brought into contact with the substrate as part of the the first indentation interval. During subsequent rotational intervals the probe is rotated over the substrate at a fixed mean vertical indentation depth, leading to friction between the probe and the substrate. During rotation, a small range of normal force variations was measured at every mean indentation position, due to the naturally occurring height variation of the surface of the hydrogel substrate. After each rotational interval, the vertical position was increased and mean normal force increased to measure friction coefficients at normal forces well below $1 N$. All measurements are highly reproducible from day to day and from probe to probe. Also, no signs of wear were observed even after repeated use of hemispherical probes and substrates. These alternating rotating and indenting intervals give us a large data set of coupled normal force $\left(F_{N}\right)$ and torque $(M)$ values at a constant preset angular velocity. With these values the dimensionless friction coefficient $(\mu)$ is determined. We verify that our custom tribometer gives the expected behavior for two dry sliding contacts $\left(F_{F}=\right.$ $\mu F_{N}$ ). The friction between a rough glass marble (Intertoys, The Netherlands) as a probe against both a commercial grade laser cut acrylic glass disk and a polystyrene foam surface as substrates was measured to validate our method (Fig. 2). For these dry solids the friction

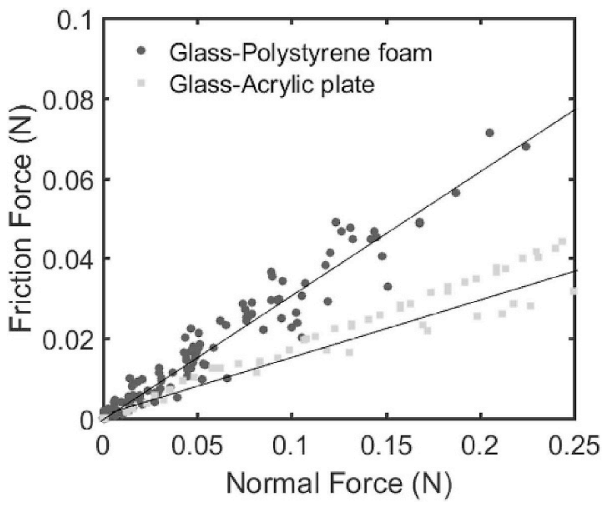

Fig. 2. Friction force - normal force curve of friction between a glass probe and polystyrene foam and between a glass probe and acrylic glass as measured with our custom-made tribotool. We observe the expected Amontons-Coulombic behavior in this context (solid lines).

force scales linearly with the normal force as is expected from Amontons-Coulomb frictional laws.

\section{Results and discussion}

To show the relevance of surface roughness in frictional studies, we designed randomly patterned rough hydrogel surfaces by molding substrate hydrogel surfaces in a mold created with sandpaper. Sandpaper comes in ranges of roughness types varying in particle height, diameter, shape and distribution which allows for a systematic variation of the hydrogel roughness (Fig. 4). Grit sizes used here are 8.4, 35, 82, 125 and $201 \mu \mathrm{m}$. We measure friction using probes and substrates made of physically cross-linked gelatin (uGel) and chemically treated irreversibly cross-linked gelatin (xGel). Chemically crosslinking the gelatin samples gives a five-fold increase of the Young's Modulus from about 0.1 MPa to 0.5 MPa. To study the effect of normal force on the friction coefficient of gelatin hydrogels we measure the friction force at increasing vertical displacements. This leads to normal forces up to at least $0.3 \mathrm{~N}$ for each sample as measured by the rheometer. The exact indentation depth corresponding to a specific range of normal forces depends on the mechanical properties of the materials in contact. Even though substrate and probe vary in shape and thickness, them having the same modulus suggests that we can make the approximation that the deformation is equally shared among substrate and the smooth hemispherical probe (radius $r_{p}=2 \mathrm{~cm}$ ). The deformation, $\delta_{p}$, of both the substrate and the probe is then $300 \mu \mathrm{m}$. Hertzian contact analysis then gives the contact radius $r_{c}=\sqrt{r_{p} \delta_{p}}$ to be approximately $1.7 \mathrm{~mm}$ and thus a contactarea of $9 \mathrm{~mm}^{2}$. A normal force of $0.3 \mathrm{~N}$ divided over this contact area gives a mean pressure of about $0.03 \mathrm{MPa}$ for the gelatin surfaces in contact. The curves constructed display overlapping data of five different experimental intervals each measured at a different range of normal forces (Fig. 3).

We immediately observe a decrease in slope with increasing normal force in our hydrogels submerged in water. The change in slope in Fig. 3 indicates a drop in friction coefficient with increasing normal forces, leading to the emergence of two distinct frictional regimes. Each of the regimes has a defined friction coefficient. This behavior is not in accordance with classical frictional laws, as friction in dry solid materials is expected to be normal force independent [16]. Changes in friction coefficient with increasing loads have however been reported for soft materials including gellan, polyvinyl alcohol (PVA) and poly(2-acrylamido-2-methylpropanesulfonic acid) (PAMPS) hydrogels on glass surfaces $[7,21,31]$. More recently this has also been found for hydrogelhydrogel contacts [18]. The uniqueness in our results is found in the clear transition from and distinction between two frictional regimes.

The first regime $\left(\mu_{1}\right)$ in Fig. 3 predominately contains relatively low 


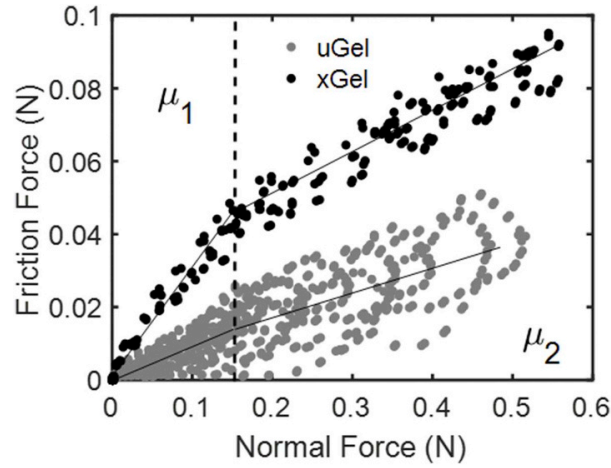

Fig. 3. Raw friction data of untreated, physically cross-linked gelatin (uGel) and chemically cross-linked gelatin (xGel) at $0.1 \mathrm{rps}$. Surfaces are patterned using sandpaper (grit size $8.4 \mu \mathrm{m}$ ). The dashed line indicates the beginning of the second frictional regime.

normal forces from 0 up to $0.15 \mathrm{~N}$ as caused by the applied indentations. The second regime $\left(\mu_{2}\right)$, after the kink, contains intervals with deeper indentations and as a result larger asperity deformations and higher normal forces. The slope of the lines represents the friction coefficient and is only moderately affected by changes in the selected starting and ending point of each regime. Selecting several different transition points in close proximity to the kink resulted in a maximum error in friction coefficient around $10 \%$.

The expression of the two regimes can be attributed to the deformable nature of the hydrogels. Applying a normal load on soft surfaces leads to deformation or even flattening of the elastic surface asperities, as was already conjectured long ago [32,33]. More recently, this deformation was also shown for two hydrogels in contact [18]. We confirm this picture through a systematic variation of the stiffness and the surface roughness of the hydrogels.

\subsection{Friction on substrates with an imposed random surface roughness}

To study the influence of material stiffness on the sudden decrease in friction coefficient found, we use surfaces made of both chemically and physically cross-linked hydrogels with polymer concentrations ranging from $10 \%$ to $20 \%$. Young's moduli ranged from 0.1 to $0.8 \mathrm{MPa}$.
We systematically vary the roughness of the hydrogels using sandpaper with different grit sizes (or average particle sizes). The asperities on the sandpaper used to make negative molds, range from very fine, densely packed grains with a grit size of $8.4 \mu \mathrm{m}$ (P2500) to coarse grains with a grit size of $201 \mu \mathrm{m}$ (P80) with large spaces between the grains and a larger size distribution of the particles. The hydrogel disks were cast on negative molds of the sandpaper to obtain surfaces with the same roughness as the sandpaper as shown in Fig. 4. We then studied the effect of stiffness as well as surface topography of hydrogels on the friction coefficient for samples with the same polymeric constituents.

With the expanded range of surface roughnesses and hydrogel moduli, we confirm the same picture: the friction coefficient is reduced with increasing normal force. The slopes in the friction force - normal force curves are used to calculate the dimensionless friction coefficient ( $\mu$ ) using $\mu=F_{F} / F_{N}$. The frictional values found for our artificially rough gelatin samples range from $\mu=0.05$ to 0.45 . These values are much lower than friction coefficients typically found for dry solids $[34,35]$ while the upper limit, $\mu=0.45$ is rather high compared to values typically reported for hydrogel-hydrogel friction using polyacrylamide or poly(2-acrylamido-2-methyl-1-propanesulfonic acid) (PAMPS) [11,18]. For gelatin substrates paired with glass, friction coefficients between 0.08 and 0.25 have been reported [36] however, the higher friction coefficients in our samples are found in the first regime and are likely caused by the imposed roughness on our hydrogel surfaces. The friction coefficients in the first regime $\left(\mu_{1}\right)$ and in the second regime $\left(\mu_{2}\right)$ for the different asperity sizes and gelatin concentrations have been summarized in Fig. 5 .

We observe that the gelatin concentration has a systematic role: gelatin tribopairs made using lower concentrations of gelatin give lower frictional values for both $\mathrm{xGel}$ and uGel (Figs. 5 and 6). The Young's moduli measured for uGel were 0.1 and $0.13 \mathrm{MPa}$ for the $15 \%$ and $20 \%$ gelatin, respectively. The Young's moduli for xGel with $10 \%$ gelatin was $0.35 \mathrm{MPa}$ while $15 \% \mathrm{xGel}$ has a modulus of $0.5 \mathrm{MPa}$ and the highest modulus was found for $20 \% \mathrm{xGel}$ with $0.8 \mathrm{MPa}$. The friction coefficients in both regimes show a logarithmic increase with the Young's modulus in both regimes. This increase is found for the smallest, most concentrated asperities (grit size $8.4 \mu \mathrm{m}$ ) up to the largest, more scattered asperities $(201 \mu \mathrm{m})$. This difference in friction coefficient can be caused by the size of the asperities (contact area) as well as the distribution of the asperities on the surface (interlocking events). The friction
$8.4 \mu \mathrm{m}(\mathrm{P} 2500)$

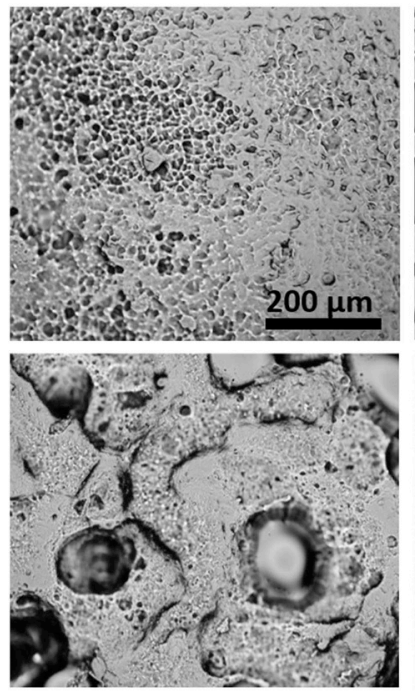

$125 \mu \mathrm{m}$ (P120)
$35 \mu \mathrm{m}(\mathrm{P} 400)$
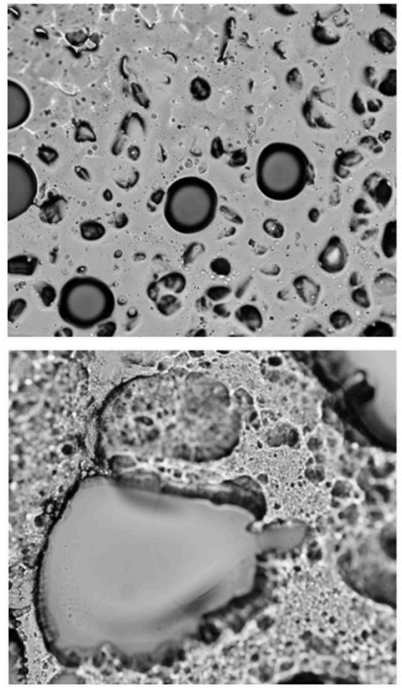

$201 \mu \mathrm{m}$ (P80)
$82 \mu \mathrm{m}(\mathrm{P} 180)$

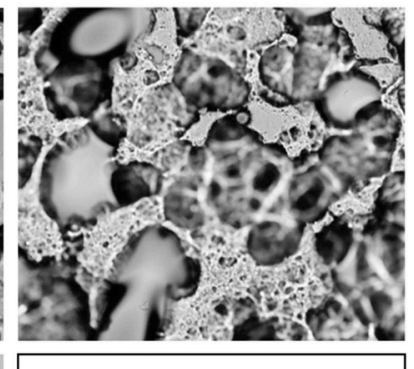

\section{DAAANANANAN}

Small dense asperities

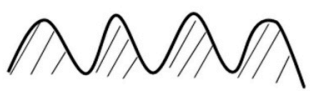

Large wide spread asperities

Fig. 4. Microscopy images of gelatin hydrogel surfaces patterned using sandpaper together with the ISO/FEPA grit size label of the sandpaper used for molding the hydrogels. The small asperities are more closely packed and have a more uniform particle size than the large asperities. 
a)
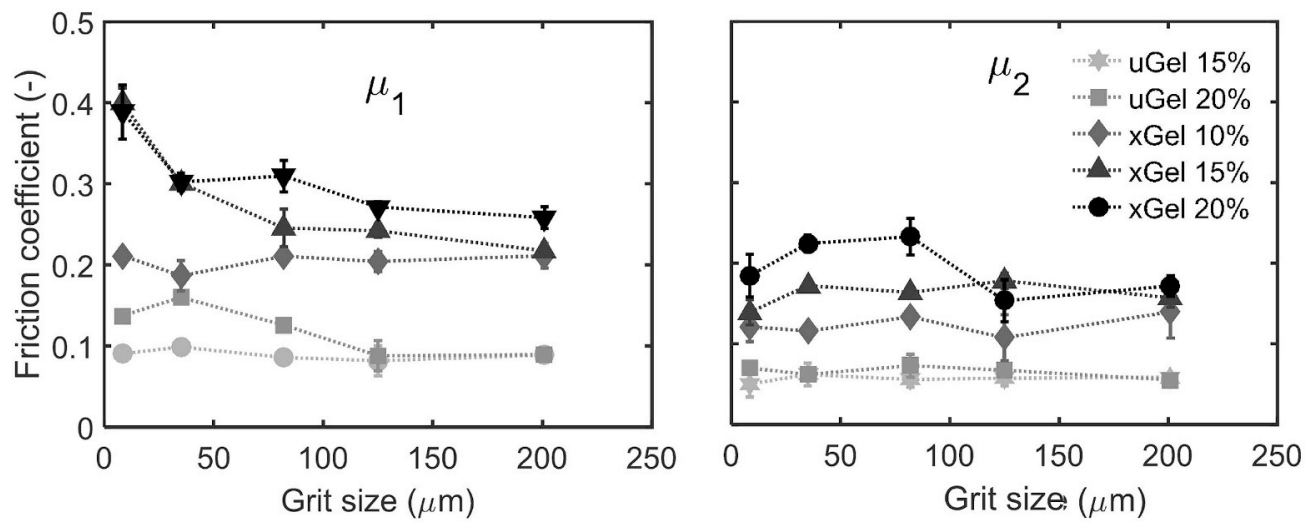

b)
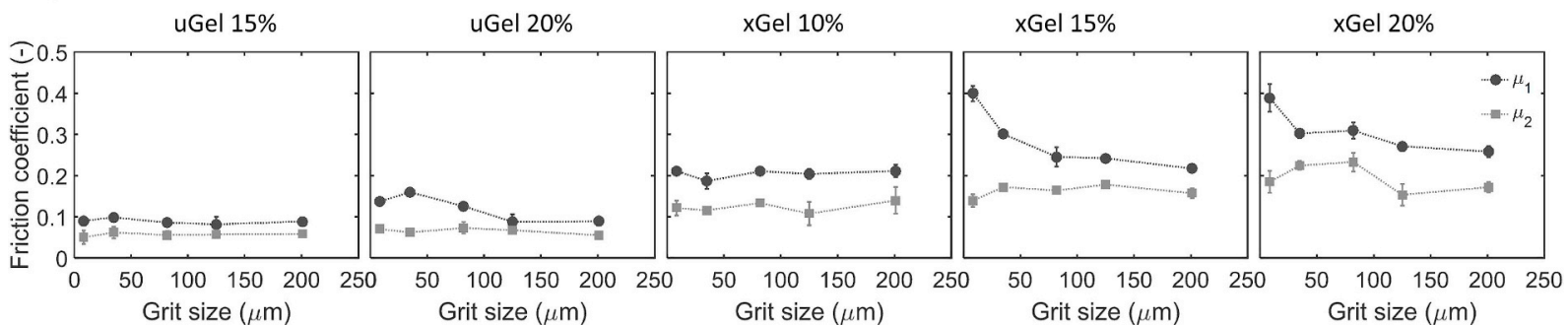

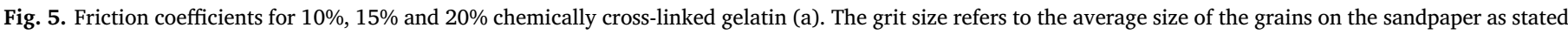

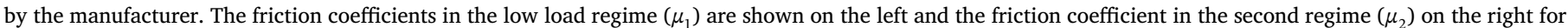

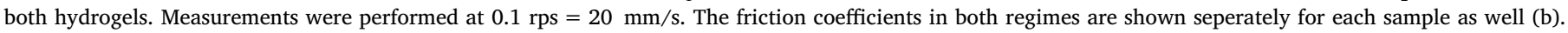

coefficient in the second regime appears independent of the grit size, and depends mainly on material stiffness. This second regime seems to be a lower limit for the friction coefficient attainable with these physically and chemically cross-linked gelatin hydrogels.

Across different polymer concentrations and Young's moduli we find a recurring trend as a function of grit size: surfaces with fine, closely packed asperities display higher friction coefficients than large asperities that are widely distributed. The stiff samples appear to be more sensitive to changes in roughness, especially below grit sizes of $100 \mu \mathrm{m}$. This thus tells us that the friction coefficient in the first regime is a result of both the asperity size and/or distribution and the Young's Modulus of the material. At low normal forces, the asperities on a rough soft surface can act as obstacles, requiring more force to move the surfaces along one another. Small asperities are expected to have more contact points and perhaps even a larger contact area than larger asperities which would explain the higher friction coefficients. The hemispherical probe also has a natural roughness scale on the order of a couple micrometers. If asperities of materials in contact are within the same size range, interlocking may occur. This in turn increases the friction between the materials [37].

As the Young's modulus of the samples increases (from left to right in Fig. 5b) the overall decrease in friction coefficient from $\mu_{1}$ to $\mu_{2}(\Delta \mu)$ becomes larger. Reducing the polymer concentration decreases the stiffness of the material which makes it easier to deform asperities leading to lower friction coefficients in the second regime (Figs. 5, 6 and 7). As the deformability decreases, the stiff asperities play an increasingly large role in hindering the probe from moving forward, resulting in high friction coefficients. Alternatively, in the low normal force regime, the asperity flattening is incomplete and probably fluctuating in time, adding a dissipation that disappears at higher normal load, where flattening effects are stronger. This effect is most pronounced at high asperity densities and small grit sizes (e.g. $8.4 \mu \mathrm{m}$ ) and may be related to the number of asperities per unit surface area.

Above $100 \mu \mathrm{m}$ interlocking events vanish as the asperities are likely too large to interlock with the surface of the hemispherical probe. In the second regime we see that the friction coefficient approaches the same $\mu_{1}$

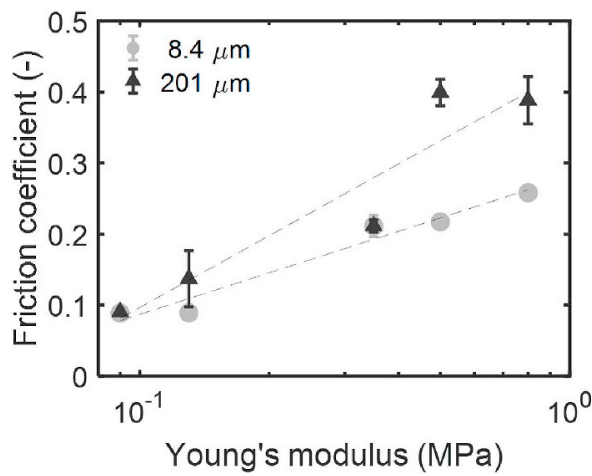

$\mu_{2}$

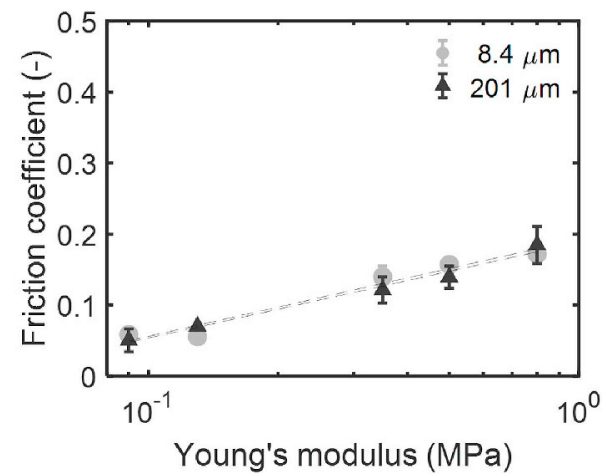

Fig. 6. The increase of the friction coefficients $\mu_{1}$ and $\mu_{2}$ as a function of Young's modulus for grit sizes 8.4 and $201 \mu m$. Hydrogels used here are $10 \%, 15 \%$ and $20 \%$ chemically cross-linked gelatin and $15 \%$ and $20 \%$ physically cross-linked gelatin at sliding velocities of $0.1 \mathrm{rps}=20 \mathrm{~mm} / \mathrm{s}$. 
a)

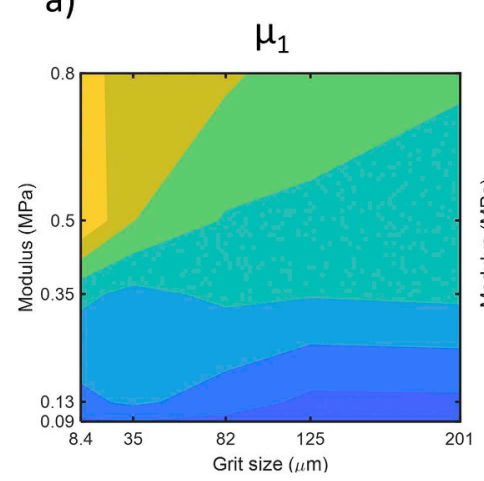

b)

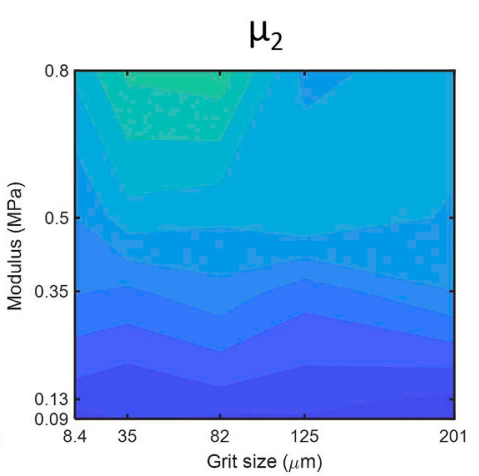

Low load

$\checkmark$

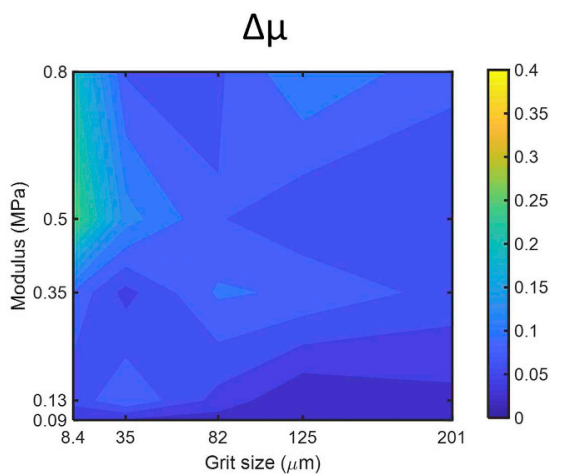

High load

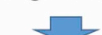

Hydrogel probe

Deformable asperities

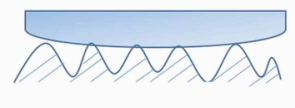

High friction

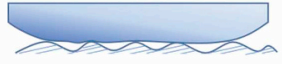

Low friction

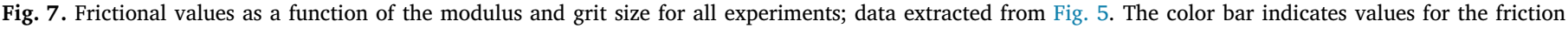

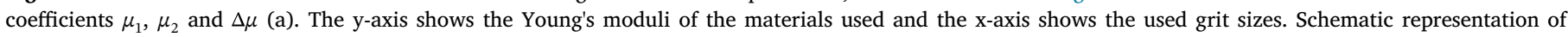

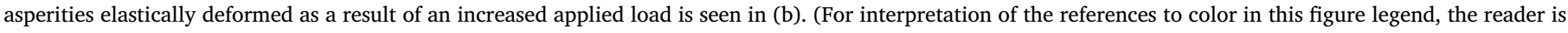
referred to the Web version of this article.)

value for each sample regardless of the roughness. This indicates that the friction coefficient in the second regime is a material property. In this low friction regime, the asperities are likely permanently flattened by the applied load (Fig. 7b) and only other dissipative dynamics (bonding/debonding, polymer entanglement, etc.) remain.

\subsection{Friction on substrates with a systematic surface roughness}

As the sandpaper surfaces are patterned randomly, the asperities vary not only in size, but also in location and distribution on the surface. To evaluate our suggested asperity-flattening mechanism, we compare regularly patterned $\mathrm{uGel}$ and $\mathrm{xGel}$ surfaces at $15 \%$ gelatin content. We specifically designed and 3D printed molds with desired asperity sizes. The distance and height of the asperities are kept constant while the diameter of the asperities is varied. The surface pillars were designed to have a height of $200 \mu \mathrm{m}$ and a distance of $500 \mu \mathrm{m}$ between the centres, so both surfaces were decorated with 4 pillars per $\mathrm{mm}^{2}$. We designed molds with two different asperity diameters; $50 \mu \mathrm{m}$ and $400 \mu \mathrm{m}$. (Fig. 8).

For $15 \%$ uGel and xGel with regularly patterned surfaces again we find a second frictional regime. $x G e l$ samples show higher friction than uGel samples (Fig. 8). Asperities with a diameter of $50 \mu \mathrm{m}$ give $\mu_{1}$ $=0.26$ and $\mu_{1}=0.11$ for $\mathrm{xGel}$ and uGel respectively, well within the range of the randomly patterned surfaces. Values in the second regime, $\mu_{2}$, approach values found for both the natural and the sandpaper patterned surfaces; 0.16 (xGel) and 0.07 (uGel) (see previous sections), which is yet more evidence for the friction coefficient in the second regime to be a material specific constant.

However, for the uGel samples with larger, $400 \mu \mathrm{m}$ pillars $\mu_{2}$ is found to be 0.17 , larger than previously found for $\mathrm{uGel}\left(\mu_{2}=0.07\right)$. For the sandpaper samples we also found the decrease in friction to be smaller for hydrogels with low moduli. This relatively small decrease in friction is likely related to the deformability of the probe and the soft pillars. Within this limit of material stiffness and asperity size, the force applied is most likely not enough to deform the relatively large $400 \mu \mathrm{m}$ pillars. As the dimensions of each asperity are now known, the compression $\Delta L$ of each individual pillar can be approximated by means of the simple relationship $\Delta L=F_{N} L / A E$, where $\Delta L$ is the deformation calculated from the normal force $F_{N}$, the initial length $L$, the area of the asperity $A$ and the Youngs

\begin{tabular}{|c|c|c|c|}
\hline & $\mu_{1}$ & $\boldsymbol{\mu}_{2}$ & $\Delta \mu$ \\
\hline xGel 50 microns & 0.26 & 0.18 & 0.08 \\
\hline xGel 400 microns & 0.32 & 0.19 & 0.13 \\
\hline uGel 50 microns & 0.11 & 0.05 & 0.06 \\
\hline uGel 400 microns & 0.22 & 0.17 & 0.05 \\
\hline
\end{tabular}
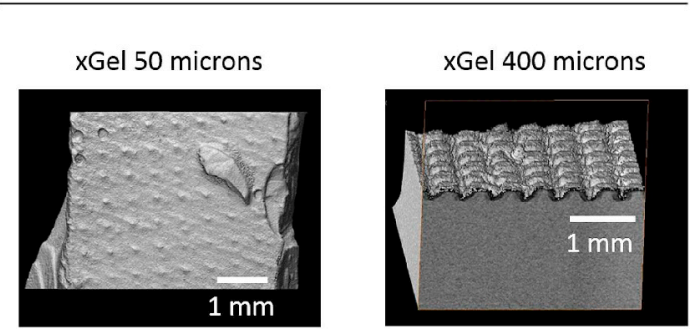

Fig. 8. Friction coefficients for patterned xGel and uGel surfaces. X-ray tomography (XRT) images of xGel with asperities of $50 \mu \mathrm{m}$ and asperities of $400 \mu \mathrm{m}$ in diameter. The asperity height and distance was kept constant at $200 \mu \mathrm{m}$ and $500 \mu \mathrm{m}$, respectively.

modulus $E$ respectively. We immediately see that the deformation for the pillars of $400 \mu \mathrm{m}$ diameter is about 60 times smaller compared to the 50 $\mu \mathrm{m}$ pillars due to the difference in aspect ratio. While the $50 \mu \mathrm{m}$ pillars can be flattened to large extent, the $400 \mu \mathrm{m}$ wide pillars experience limited compression. It appears that the regularity of the surface patterning plays an important role: irregularly patterned surfaces have a wide distribution of asperity heights. This leads to the normal force applied being distributed over the few asperities sticking out at every compression level. In contrast, the regular pillar height of the patterned rough substrate imposes that at all times, the normal force applied will be distributed over many pillars. By creating large and evenly sized pillars, the ability to flatten the asperities has been restrained, leading to a limited decrease in friction. Patterning the hydrogels in a controlled manner is thus an efficient way to tune the friction coefficient and its sensitivity to changes in normal force. 


\subsection{Friction on substrates with a natural surface roughness}

In what way does friction of samples with an imposed surface roughness resemble the behavior of hydrogel surfaces generated in smooth molds? It has been found that gellan, polyvinyl alcohol (PVA) and poly(2-acrylamido-2-methylpropanesulfonic acid) (PAMPS) cast on smooth surfaces all show different frictional behaviour when sliding against glass substrates $[7,21,31]$. This is commonly evaluated in terms of molecular interactions between the hydrogel-glass interface. However, in previous sections we have seen how important the role of surface roughness is. This makes it interesting to consider the role of the surface roughness and how the chemical composition of a hydrogel affects its surface structure.

We use four hydrogels with varying network structures: physically cross-linked gelatin (uGel), chemically cross-linked gelatin (xGel), agar and polyacrylamide (PAAm). Gelatin is known to form a triple helical structure while agar forms double helices [38,39]. PAAm, a synthetic polymer, forms short and rigid cross-links due to the presence of bisacrylamide chains that cross-link the longer polymer strands [40]. Due to the differences in network formation and polymer concentration, we obtain gels with different moduli. Physically cross-linked gelatin provides the lowest modulus (0.1 MPa), followed by agar ( $0.2 \mathrm{MPa})$, and PAAm (0.5 MPa) and chemically cross-linked gelatin (0.5 MPa).

It turns out that the chemical structure of the gel affects not only the bulk properties of the hydrogels, but also their surface properties. To differentiate between the different degrees of naturally occurring surface roughness, we performed cryo-SEM imaging on the hydrogel surfaces (Fig. 9). The top surfaces of the hydrogels, on which we also perform tribological test (see below), are seen above the dashed lines; the internal gel structures are visible below the line. In addition, we show the schematic network structure that is typically expected for these hydrogels.

The PAAm hydrogel (Fig. 9a) with short and linear chemical crosslinks has the smoothest surface according to our cryo-SEM results. The lack of structure in the assembling molecules gives the gel a tightly bound structure resulting in a flat surface. Agar (Fig. 9b) and gelatin hydrogels show a rougher surface (Fig. 9c and d). This relatively rough surface may be caused by coil-like helical structures that build up the hydrogels $[38,39]$. The covalent chemical cross-links formed by glutaraldehyde transform the wavy gelatin surface (c) into a rather bumpy landscape (d). The chemical cross-linker irreversibly brings polymer chains closer together leading to a stiffer network which apparently has a higher degree of roughness.

By varying the polymer constituents and the interactions between them, we have obtained four hydrogels with distinct bulk and surface properties. Fig. 10 shows the results of the lubricated friction between physically cross-linked or untreated gelatin (uGel), chemically cross- linked gelatin (xGel), agar and polyacrylamide (PAAm) tribopairs in water. The values of the friction coefficients in both frictional regimes $\left(\mu_{1}\right.$ and $\left.\mu_{2}\right)$ and the difference between the two regimes $(\Delta \mu)$ together with the Young's moduli (E) of the hydrogels are also shown here. Each hydrogel has a distinct friction coefficient that can be related to the degrees of roughness observed in the cryo-SEM images (Fig. 9).

The frictional behavior of these diverse samples further demonstrate that the normal force dependency is a robust property of hydrogel friction. Interestingly, the decrease in friction is absent in dry, solid friction experiments and extremely low for lubricated PAAm friction. This is likely because the asperities in dry solids are too stiff to be deformed at the normal forces used here. The PAAm surface is clearly smoother than that of the remaining hydrogels, with no irregularities to be deformed in a second regime.

The different hydrogels submerged in water give low friction coefficients in a range from 0.008 to 0.31 . In the second regime, $\mathrm{xGel}$ shows the highest friction coefficient followed by $\mathrm{uGel}$ and agar. PAAm has the lowest friction coefficient in this regime. Samples made of $x \mathrm{Gel}$ and PAAm have similar Young's moduli (0.5 MPa), yet we see large differences in the friction coefficients of these two hydrogels (Fig. 10). The low friction coefficient found for PAAm in the first regime can be related to the smooth surface as observed by cryo-SEM (Fig. 9): there are no asperities to flatten and the molecular structure of PAAm apparently does not give rise to other dissipative mechanisms, producing a dissipation during sliding that is only borderline detectable in our setup. The hydrogels with a more pronounced surface roughness all show two regimes of friction and always higher friction coefficients than PAAm. As seen in previous sections, hydrogel-hydrogel tribology indeed depends on a combined effect of bulk and surface properties.

\subsection{The role of viscous or polymeric lubrication}

Physical mechanisms that are commonly discussed in hydrogel friction include the presence of a fluid layer [19], gel relaxation [9,28], poroelastic effects [17,41], and surface adhesion [26]. These mechanisms are potentially also involved in the decrease of friction we find in our hydrogels. As our measurements are carried out in water, we do not expect adhesion between the hydrogels to be of large influence. A thin film of water being squeezed out of the hydrogel is not expected to significantly further decrease the friction, as measurements are carried out in large amounts of water and a fluid layer is already present [7]. In addition, the asperities of rough gels would expel more water than flat gels under an applied load as the asperities undergo a large compression [19]. This would lead to a larger decrease in friction for rough gels, which we do not observe in our measurements.

We look further into the drop in friction coefficient by performing
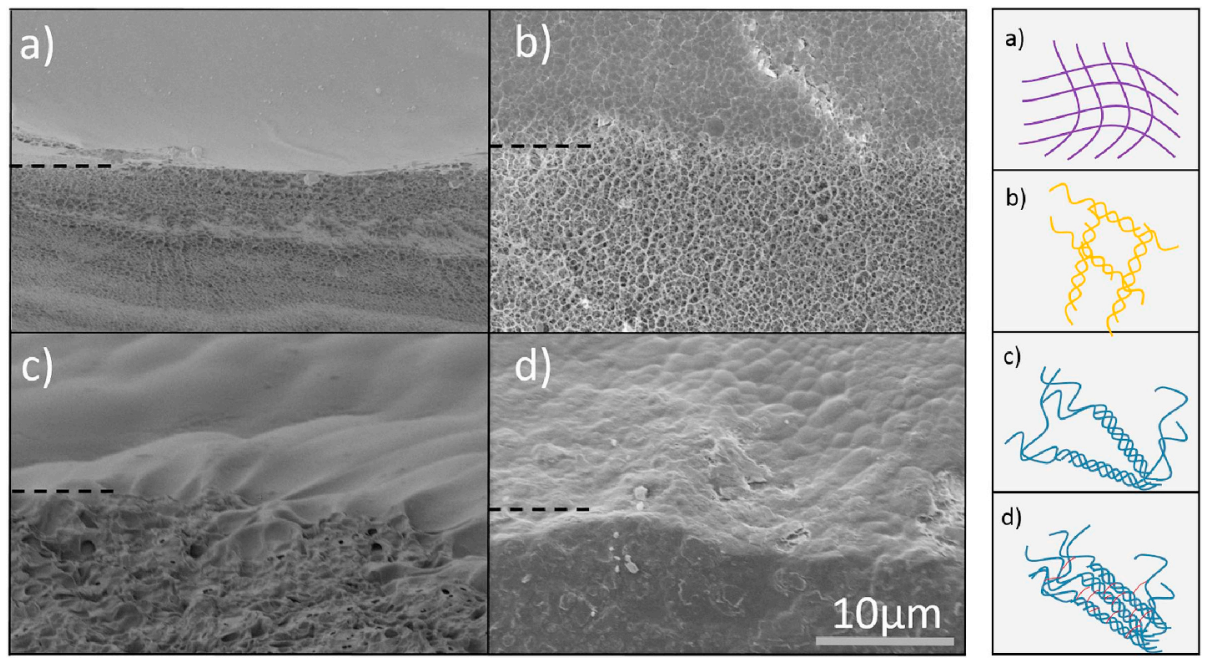

Fig. 9. Cryo-SEM images of the surface and internal structures of PAAm (a), agar (b), uGel (c) and xGel (d) on the left with the proposed schematic polymer network structure shown on the right. Microscope images were obtained using scanning electron microscopy with a magnification of 8000 times at cryogenic temperatures. North of the dashed line represents the surface. Below the dashed line the internal structure of the hydrogels can be seen. 


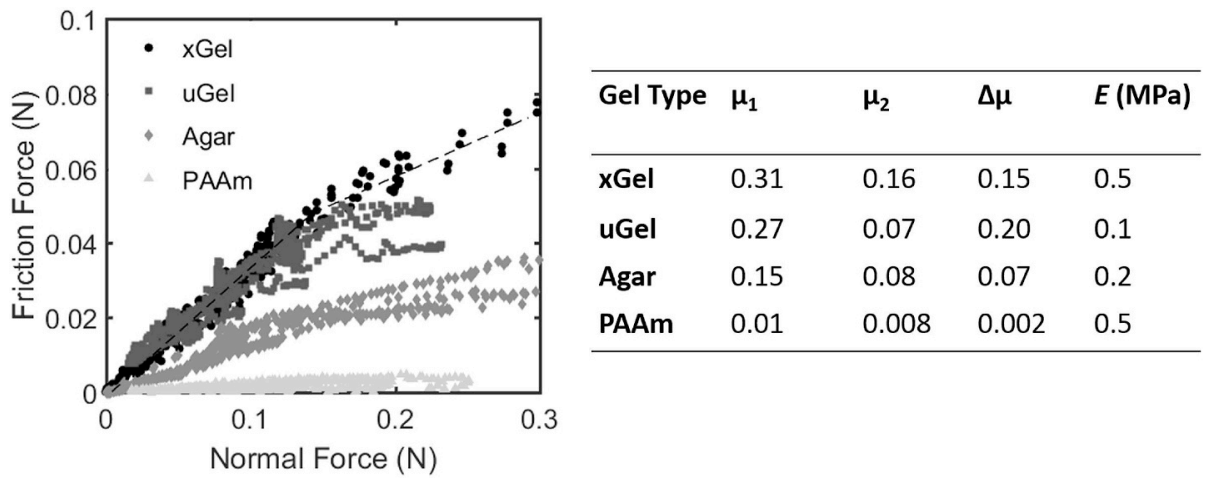

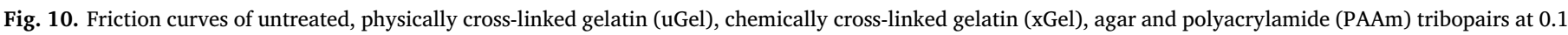

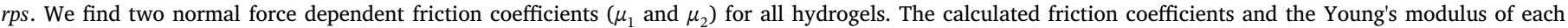
sample are shown in the table on the right.

measurements with longer duration and lower sliding velocities, to determine whether time or velocity dependent effects are at play in hydrogel-hydrogel friction. For this purpose we selected xGel, as this chemically cross-linked material is stable over a long period of time with respect to swelling. Since the cross-linking is performed in a waterbath with glutaraldehyde and the gel is then immediately submerged and stored in water, maximum swelling is already attained. The results in Table 1 show that the friction between two cross-linked gelatin hydrogels are time and velocity independent within the available velocity range of our equipment. Friction coefficients $\mu_{1}$ and $\mu_{2}$ for xGel remained constant within the measuring range of $0.005-0.1 \mathrm{rps}$. The rate and time independence found for $\mathrm{xGel}$ indicates that the decrease in friction is indeed not caused by adhesion or fluid film formations at the interface as these are rate-dependent phenomena. We also do not observe any significant wear effects in these soft materials. Frictional measurements performed on the same substrate and probe pairs consistently yielded reproducible results over several weeks.

Time-dependent effects such as fluid migrating to the interface, relaxation phenomena, or serum release, do not seem to be the main driving forces behind the normal force dependence found for hydrogels. Another effect leading to a decrease in friction in hydrogels might be the loosening or dissolving of polymers within the hydrogel network [7,31]. A viscous layer could be formed on the hydrogel interface which promotes a decrease in friction. In chemically cross-linked substrates this effect would be less profound as the polymers are covalently bound. However, we do find a large drop in friction coefficient in our chemically cross-linked $x \mathrm{Gel}$, eliminating loosening of polymer layers as the main cause for the normal force dependence. Repeating a full cycle of measurements with the same samples yields highly reproducible results. Loosening or dissolving polymer chains in a permanent manner is thus an unlikely source of reduced friction in the second regime.

\subsection{Discussion}

With the combined results of the imposed regular, irregular and naturally rough surfaces it is clear that elastic deformation of the surface asperities plays a key role in the observed drop in friction

Table 1

Friction coefficients for xGel at $0.1,0.05,0.001$ and 0.005 rps in the low-load $\left(\mu_{1}\right)$ and the high-load $\left(\mu_{2}\right)$ regime. The measurements at different velocities were carried out with the same hydrogel probe and substrate.

\begin{tabular}{llc}
\hline Angular velocity (rps) & $\mu_{1}$ & $\mu_{2}$ \\
\hline $\mathbf{0 . 1}$ & 0.31 & 0.16 \\
$\mathbf{0 . 0 5}$ & 0.33 & 0.16 \\
$\mathbf{0 . 0 1}$ & 0.28 & 0.14 \\
$\mathbf{0 . 0 0 5}$ & 0.28 & 0.15 \\
\hline
\end{tabular}

coefficient. This is concretely observed by making direct comparisons: we find that for materials with the same stiffness (xGel and PAAm) the difference in their surface roughness leads to a large difference in friction coefficients in both regimes. When comparing two relatively rough materials (xGel and $\mathrm{uGel}$ ), our results demonstrate that the stiffness plays an increasingly large role. The appearance of a second frictional regime at high normal forces thus appears to be a function of the stiffness and surface roughness (imposed or induced by the polymeric nature or the hydrogel) regardless of the length scale of the asperities. Further parameters contributing to the friction coefficients of hydrogels include the difference in chemical make-up along with the mesh sizes and network structures for each of the samples.

We can further elucidate the asperity flattening hypothesis by estimating the asperity deformation at a normal force $F_{N}$ of $0.2 \mathrm{~N}$ and maximum probe displacement of $400 \mu \mathrm{m}$. The contact area is then approximately $6 \mathrm{~mm}^{2}$ as estimated using Hertz theory. The hardest gel surface (xGel) has asperities with a radius of roughly $r_{a}=1.5 \mu \mathrm{m}$ as estimated from Fig. 9. The number of asperities with which the probe is in contact is then approximately $2 \times 10^{6}$ if we assume all asperities are hemispherical in shape and completely cover the surface. Every asperity thus experiences a contact force of $\sim 1 \times 10^{-7} \mathrm{~N}$. To estimate the deformation of a single naturally occurring asperity, we assume that every single substrate asperity equally exerts $1 \times 10^{-7} \mathrm{~N}$ on the probe, which due to the separation of length scales can be considered a flat plane. We would like to again highlight that this is an underestimation: due to the irregularity of the surface, the applied normal load is most likely not evenly distributed over all contacts, but instead focused on only a few asperities. We then estimate the deformation of an asperity under such load again with Hertz theory, $F_{a}=\frac{4}{3} E r_{a}^{1 / 2} \delta_{a}^{3 / 2}$ to be $\delta_{a} / r_{a} \sim 0.1$. In this limit the deformation predicted by Hertz theory is already ten percent of the asperity radius. This is in fact independent of $r_{a}$ : the relative deformation of an asperity is $\delta_{a} / r_{a}=\frac{3 F_{N}}{4 E \delta_{p} r_{p}}$. This supports our hypothesis that deformation of asperities plays a significant role, even for the stiffest gels used. The less stiff gels are easier to deform or flatten and therefore give an even larger drop in the friction coefficient in the second regime. This estimation gives a clear suggestion of the physical mechanisms behind the asperity flattening. However, it does not include the height of the asperities which can not be estimated from the cryo-SEM images. The spatial variability of the pressure in the Hertz solution, a possible deviation from incompressibility and deformation coupling between asperities have been omitted from the estimation for simplicity. Recently it was found in "Gemini" hydrogel pairs, that an increase in normal force leads to a decrease in friction in the speed independent regime [18]. Hertzian contact mechanical behavior was observed for these hydrogels and the contact area, friction coefficient and normal force were found to be directly related to one another $(\mu \alpha$ $F_{N}^{-1 / 3}$ ). As we have shown here, Hertzian behavior alone would not suffice to accurately describe the two distinct regimes observed in 
hydrogel friction with our rough substrates.

\section{Conclusions}

Friction on soft substrates is influenced by many factors including surface properties such as roughness, and bulk properties such as the elasticity. We measure friction on a range of patterned hydrogels with different chemical compositions using a customized tribotool. The frictional dynamics observed are strongly normal force dependent in our soft lubricated contacts. When increasing the normal force, a drop in friction coefficient is found, defining two distinct frictional regimes. The first frictional regime is sensitive to the nature and the length scale of the asperities (synthetic or naturally occurring) on the hydrogel surface. The friction coefficient in the second regime appears to be a material property. For samples made of the same polymeric materials, we find that lower Young's moduli lead to lower friction in both regimes regardless of the surface roughness. The decrease in friction coefficient is likely to be induced by the deformation of the soft surface asperities leading to a smoother surface. In the second regime, dissipative dynamics are determined by other bulk or surface properties.

The experimental tribology method described here enables to explore the complexity behind lubricated hydrogel-hydrogel friction over a wide range of material types, surface characteristics and lubricants. It is evident from our results that both hydrogel material properties and the topography of the surface are of importance when rationally designing surfaces with specific frictional properties. Our observations clarified the role of substrate hardness and surface asperities even in seemingly smooth surfaces. The regularly patterned hydrogel surfaces that we introduced point towards hydrogels as being perfect candidates for different applications when low friction coefficients and easily accessible control parameters are desired. These patterned materials can ultimately be used for applications such as biomedical devices or food materials with specific textural features.

\section{Acknowledgements}

The authors thank Marcel Workamp and Pieter de Visser for their help in designing and operating the 3D printed tribotool, Jonathan Barés for providing the first sketch of the hemispherical cap mold, Hannie van den Broek for suggesting to perform, and providing assistance with cryo-SEM measurements, and other microscopy support and Remco Hamoen for support with XRT measurements. Research presented in this article was financially supported by the Graduate School VLAG.

\section{References}

[1] Milner PE, Parkes M, Puetzer JL, Chapman R, Stevens MM, Cann P, Jeffers JR. A low friction, biphasic and boundary lubricating hydrogel for cartilage replacement. Acta Biomater 2018;65:102-11.

[2] Karimi A, Navidbakhsh M. Material properties in unconfined compression of gelatin hydrogel for skin tissue engineering applications. Biomed Eng Biomed Tech 2014;59:479-86.

[3] Ma J, Lee J, Han SS, Oh KH, Nam KT, Sun J-Y. Highly stretchable and notch-insensitive hydrogel based on polyacrylamide and milk protein. ACS Appl Mater Interfaces 2016;8:29220-6.

[4] Shewan HM, Stokes JR. Review of techniques to manufacture micro-hydrogel particles for the food industry and their applications. J Food Eng 2013;119:781-92.

[5] Gomez-Guillen MC, Gimenez B, Lopez-Caballero ME, Montero MP. Functional and bioactive properties of collagen and gelatin from alternative sources: a review. Food Hydrocolloids 2011;25:1813-27.

[6] Torres O, Reyes EA, Murray BS, Sarkar A. Emulsion microgel particles as high performance bio-lubricants. ACS Appl Mater Interfaces 2018;10(32):26893-905. https://doi.org/10.1021/acsami.8b07883.

[7] Gong JP, Iwasaki Y, Osada Y. Friction of gels. 5. negative load dependence of polysaccharide gels. J Phys Chem B 2000;104:3423-8.

[8] Liu G, Liu Z, Li N, Wang X, Zhou F, Liu W. Hairy polyelectrolyte brushes-grafted thermosensitive microgels as artificial synovial fluid for simultaneous biomimetic lubrication and arthritis treatment. ACS Appl Mater Interfaces 2014;6:20452-63.

[9] Pitenis AA, Uruena JM, Schulze KD, Nixon RM, Dunn AC, Krick BA, Sawyer WG, Angelini TE. Polymer fluctuation lubrication in hydrogel gemini interfaces. Soft Matter 2014;10:8955-62.

[10] Tominaga T, Takedomi N, Biederman H, Furukawa H, Osada Y, Gong JP. Effect of substrate adhesion and hydrophobicity on hydrogel friction. Soft Matter 2008;4:1033-40.

[11] Gong JP, Osada Y. Surface friction of polymer gels. Prog Polym Sci 2002;27:3-38.

[12] Rudge RED, Scholten E, Dijksman JA. Advances and challenges in soft tribology with applications to foods. Curr Opin Food Sci 2019;27:90-7.

[13] Skotheim J, Mahadevan L. Soft lubrication. Phys Rev Lett 2004;92:245509.

[14] Leroy S, Steinberger A, Cottin-Bizonne C, Restagno F, Léger L, Charlaix É. Hydrodynamic interaction between a spherical particle and an elastic surface: a gentle probe for soft thin films. Phys Rev Lett 2012;108:264501.

[15] Sekimoto K, Leibler L. A mechanism for shear thickening of polymer-bearing surfaces: elasto-hydrodynamic coupling. Europhys Lett 1993;23:113.

[16] Amontons G. Remarques et experiences phisiques sur la construction d'une nouvelle clepsidre, sur les barometres, termometres, \& higrometres, chez Jean Jombert. 1695. Paris.

[17] Dolan GK, Yakubov GE, Bonilla MR, Lopez-Sanchezb P, Stokes JR. Friction, lubrication, and in situ mechanics of poroelastic cellulose hydrogels. Soft Matter 2017; 13:3592-601.

[18] Urueña JM, McGhee EO, Angelini TE, Dowson D, Sawyer WG, Pitenis AA. Normal load scaling of friction in Gemini hydrogels. Biotribology 2018;13:30-5.

[19] Yashima S, Takase N, Kurokawa T, Gong JP. Friction of hydrogels with controlled surface roughness on solid flat substrates. Soft Matter 2014;10:3192-9.

[20] Riedo E, Brune H. Young modulus dependence of nanoscopic friction coefficient in hard coatings. Appl Phys Lett 2003;83:1986-8.

[21] Gong JP. Friction and lubrication of hydrogels - its richness and complexity. Soft Matter 2006;2:544-52.

[22] Dunn AC, Sawyer WG, Angelini TE. Gemini interfaces in aqueous lubrication with hydrogels. Tribol Lett 2014;54:59-66.

[23] Drozdov A, Sanporean C-G, Christiansen J. Mechanical response of HEMA gel under cyclic deformation: viscoplasticity and swelling-induced recovery. Int J Solids Struct 2015;52:220-34.

[24] Zhao X. Multi-scale multi-mechanism design of tough hydrogels: building dissipation into stretchy networks. Soft Matter 2014;10:672-87.

[25] Gong JP, Kagata G, Iwasaki Y, Osada Y. Surface dynamic friction of polymer gels. Chin J Polym Sci 2000;18:271-5.

[26] Gong J, Iwasaki Y, Osada Y, Kurihara K, Hamai Y. Friction of gels. 3. friction on solid surfaces. J Phys Chem B 1999;103:6001-6.

[27] Dabrowska A, Rotaru GM, Spano F, Affolter C, Fortunato G, Lehmann S, Derler S, Spencer ND, Rossi RM. A water-responsive, gelatine-based human skin model. Tribol Int 2017;113:316-22.

[28] Urueña JM, Pitenis AA, Nixon RM, Schulze KD, Angelini TE, Sawyer WG. Mesh size control of polymer fluctuation lubrication in Gemini hydrogels. Biotribology 2015;1:24-9.

[29] Kim J-Y, Song J-Y, Lee E-J, Park S-K. Rheological properties and microstructures of carbopol gel network system. Colloid Polym Sci 2003;281:614-23.

[30] Workamp M, Dijksman JA. Contact tribology also affects the slow flow behavior of granular emulsions. J Rheol 2019;63:275-83.

[31] Kim SH, Opdahl A, Marmo C, Somorjai GA. Afm and SFG studies of pHEMA-based hydrogel contact lens surfaces in saline solution: adhesion, friction, and the presence of non-crosslinked polymer chains at the surface. Biomaterials 2002;23:1657-66.

[32] Denny D. The influence of load and surface roughness on the friction of rubber-like materials. Proc Phys Soc Sect B 1953;66:721.

[33] Schallamach A. Friction and abrasion of rubber. Rubber Chem Technol 1958;31:982-1014.

[34] Blau PJ. The significance and use of the friction coefficient. Tribol In 2001;34:585-91.

[35] Lorenz B, Oh Y, Nam S, Jeon S, Persson B. Rubber friction on road surfaces: experiment and theory for low sliding speeds. J Chem Phys 2015;142:194701.

[36] Baumberger T, Caroli C, Ronsin O. Self-healing slip pulses and the friction of gelatin gels. Eur Phys J E 2003;11:85-93.

[37] Müser MH, Wenning L, Robbins MO. Simple microscopic theory of Amontons's laws for static friction. Phys Rev Lett 2001;86:1295.

[38] Duconseille A, Andueza D, Picard F, Santé-Lhoutellier V, Astruc T. Molecular changes in gelatin aging observed by nir and fluorescence spectroscopy. Food Hydrocolloids 2016;61:496-503.

[39] Taylor MJ, Tomlins P, Sahota TS. Thermoresponsive gels. Gels 2017;3:4.

[40] Baek K, Clay NE, Qin EC, Sullivan KM, Kim DH, Kong H. In situ assembly of the collagen-polyacrylamide interpenetrating network hydrogel: enabling decoupled control of stiffness and degree of swelling. Eur Polym J 2015;72:413-22.

[41] Reale ER, Dunn AC. Poroelasticity-driven lubrication in hydrogel interfaces. Soft Matter 2017;13:428-35. 